\title{
A COMPARATIVE STUDY OF LAPAROTOMY WOUNDS CLOSED WITH INTERRUPTED-X TECHNIQUE AND CONVENTIONAL CONTINUOUS CLOSURES WITH VICRYL
}

\author{
Sanjeev Sharma1, B. L. Sunkaria², Gagandeep Singh ${ }^{3}$ \\ ${ }^{1}$ Associate Professor, Department of General Surgery, Government Medical College, Amritsar. \\ ${ }^{2}$ Associate Professor, Department of General Surgery, Government Medical College, Amritsar. \\ 3 Junior Resident, Department of General Surgery, Government Medical College, Amritsar.
}

\section{ABSTRACT}

\section{BACKGROUND}

This study is important in lieu of one of the most important complications associated with exploratory laparotomy i.e. burst abdomen or abdominal wound dehiscence, which is highly linked to the high morbidity and mortality of the diseased.

\section{MATERIALS AND METHODS}

40 patients were randomised to have the abdominal wall closed by interrupted-X technique and conventional continuous layered closure into two groups equally (group 1 and group 2 respectively).

\section{RESULTS}

In interrupted-X group, wound dehiscence rate was found to be $15 \%$ as compared to $25 \%$ in the continuous group. Incidence of burst abdomen/incisional hernia in the two groups was $5 \%$ and $15 \%$ respectively at the end of 3 months.

\section{CONCLUSION}

Interrupted-X technique was associated with decreased risk of wound dehiscence, burst abdomen and incisional hernia. Interrupted-X closure should be the preferred method of closure for abdominal fascia in exploratory laparotomies as there is decreased propensity for development of wound dehiscence, burst abdomen and incisional hernia with it.

\section{KEYWORDS}

Interrupted-X Technique, Conventional Continuous Layered Closure, Exploratory Laparotomy, Abdominal Wound Dehiscence, Burst Abdomen, Incisional Hernia.

HOW TO CITE THIS ARTICLE: Sharma S, Sunkaria BL, Singh G. A comparative study of laparotomy wounds closed with interrupted-X technique and conventional continuous closures with Vicryl. J. Evolution Med. Dent. Sci. 2017;6(21):1710-1713, DOI: $10.14260 /$ Jemds/2017/376

\section{BACKGROUND}

Since ages, there has been an argumentation about the ideal method of closure of midline vertical abdominal incision following a laparotomy. The search for an ideal method of abdominal wound closure has been long ongoing. $1,2,3$ The techniques are modified frequently but the goal of wound closure has always remained the same i.e. to restore function of abdominal wall after the surgical procedure. This discussion is important because one common complication of laparotomy is burst abdomen or abdominal wound dehiscence, which is associated with high morbidity, mortality, and cost of care. Numerous studies have been carried out for evaluating different closure techniques. Western studies project no compelling differences in the risk of burst between continuous and interrupted methods of closure. ${ }^{4,5}$ But in a developing country like India where most of these patients are malnourished and present with intraperitoneal sepsis, the search for an optimal and safest method for closure becomes of utmost importance.

Financial or Other, Competing Interest: None.

Submission 02-02-2017, Peer Review 01-03-2017,

Acceptance 07-03-2017, Published 13-03-2017.

Corresponding Author:

Dr. Gagandeep Singh,

\#r/o 562-B, Palam Vihar,

Pakhowal Road,

Ludhiana-142022.

E-mail:dr.gagan1983@gmail.com

DOI: $10.14260 /$ jemds $/ 2017 / 376$
The ideal method should be technically simple and it should leave the patient with a reasonably aesthetic scar and most importantly, it should minimise the frequency of wound rupture, incisional hernia (IH), wound infection and sinus formation.

The study was conducted in the Department of Surgery, Government Medical College and Guru Nanak Dev Hospital, Amritsar. The study included 40 patients, who were divided into two groups. The aims of the study were-

1. To measure the risk of abdominal wound dehiscence for the above two techniques i.e. conventional continuous and interrupted-X methods.

2. To calculate the relative risk of burst between conventional continuous and interrupted-X methods.

3. Compare the post-operative complications of laparotomy wounds like seroma, wound infection, wound gaping, burst abdomen and incisional hernia in the two groups.

\section{MATERIALS AND METHODS \\ Source of Data}

40 patients admitted in the Department of Surgery, GMC Amritsar, were included in the study. The patients were chosen randomly, irrespective of gender, age and nature of disease. Out of these 40 patients, 20 were randomised to have the abdominal wall closed by interrupted-X technique and remaining 20 by conventional continuous layered closure and they were grouped as group 1 and group 2 respectively. 


\section{Method of Data Collection}

History regarding the particular illnesses was noted in each patient followed by clinical examination and the routine investigations like blood and urine. Other necessary investigations were sent for. Special investigations like ultrasonography or CT scan, relevant to the disease were done.

\section{Inclusion Criteria}

- $\quad$ Patients aged 15-75 years.

- Patients posted for laparotomy in an emergency setup.

- Patients who underwent laparotomy with midline incisions.

\section{Exclusion Criteria}

- Patients with comorbid conditions like diabetes mellitus, immune compromised patients, patients on cancer chemotherapy, immunotherapy and on longterm steroids.

- $\quad$ Patients who died within 7 days after surgery.

- Patients who underwent surgery by Grid-iron and Transverse abdominal incisions.

- Patients who underwent second laparotomy or relaparotomy.

\section{Investigations or Interventions required to be conducted} on Patients if so are Briefly Described Below

1. Blood

- Haemoglobin.

- Total count.

- Differential count.

- Random blood glucose.

- Blood urea, serum creatinine.

- Serum electrolytes.

2. Urine routine.

3. ECG.

4. Chest X-ray PA view.

5. Plain X-ray abdomen in erect posture.

6. Contrast X-rays like barium meal wherever applicable.

7. Upper GI endoscopy wherever applicable.

8. Abdominal ultrasound and CT scan wherever applicable.

9. Wound swab culture and sensitivity in case of surgical site infection if any.

\section{Suturing Techniques Compared}

Written informed consent was taken from all the patients. Patients were subsequently divided into the following two groups for closure by random allotment.

\section{Interrupted Double- X Closure}

It was performed using No. 1 Vicryl suture. A large bite to be taken on cut edge of linea alba from outside-in, $2 \mathrm{~cm}$ from the edge. The needle will emerge on the other side from insideout diagonally $2 \mathrm{~cm}$ from the edge and $4 \mathrm{~cm}$ above or below the first bite. This strand will be crossed or looped around the free end of suture and continued outside-in, diagonally at $90^{\circ}$ to the first diagonal. The two ends will be tied just tight enough to approximate the edges of linea alba taking care not to include bowel or greater omentum between the edges. The small free end of the suture will be passed deep to the $\mathrm{X}$ behind linea alba and again tied to the other end of the suture. This method of tying four throws in front and four throws behind the $\mathrm{X}$ will create two X-like crosses: one on the surface and other on the deep to linea alba. The central knot will allow fixation of four arms of the $\mathrm{X}$ like a pivot. The next $\mathrm{X}$ suture will be placed $1 \mathrm{~cm}$ away (above or below) from the previous one. Thus, in a $14 \mathrm{~cm}$ long wound, $3 \mathrm{X}$-sutures will be applied. The suture line will then be palpated for any gap with the index finger. Any large gap permitting a finger will be closed with a simple interrupted suture.
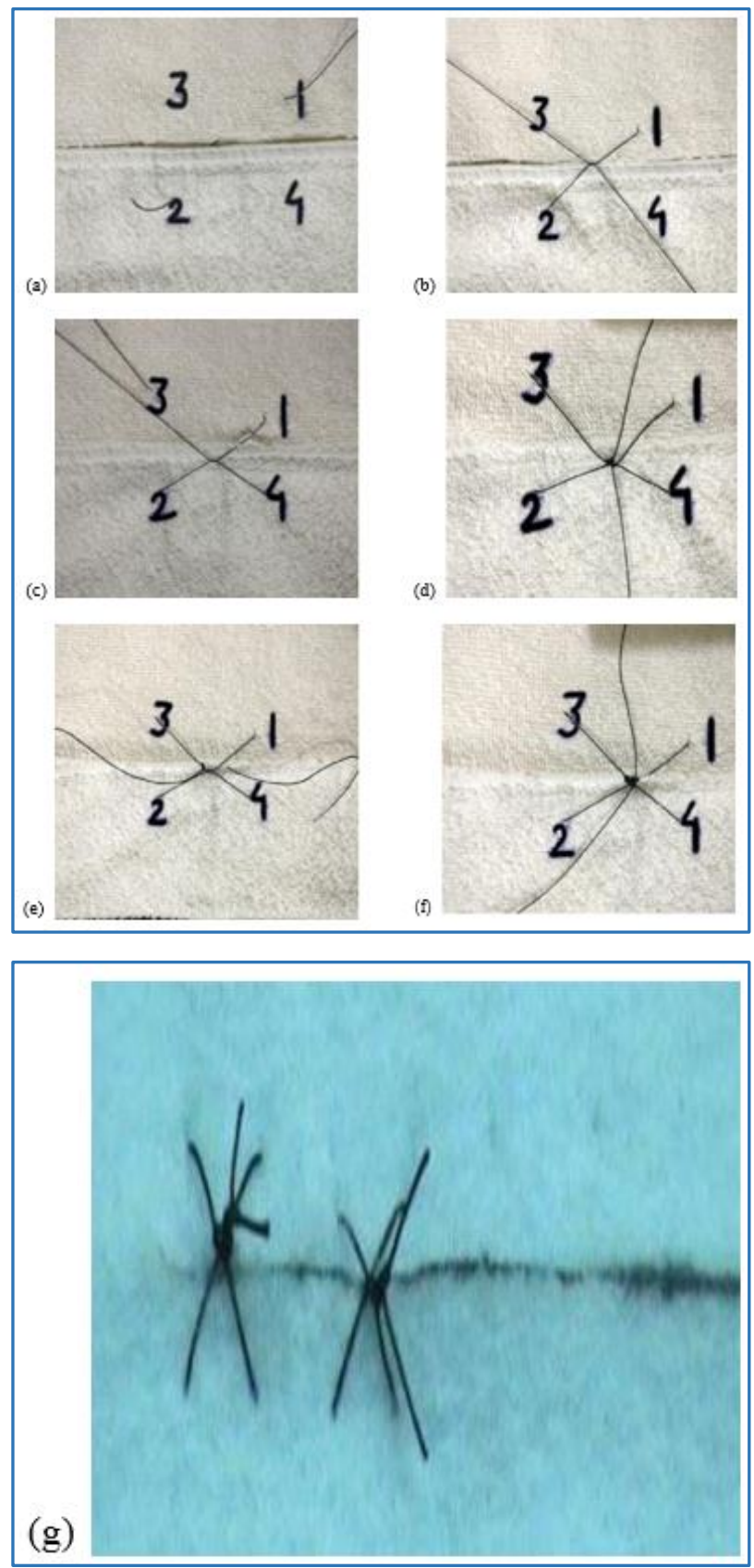

Figure 1. Interrupted $X$-suture

a) A bite is taken at (1) a point $2 \mathrm{~cm}$ from cut edge. The needle emerges at (2) another point $2 \mathrm{~cm}$ from cut edge, $4 \mathrm{~cm}$ cranial or caudal to (1).

b) The 2 ends of suture strand crossed.

c) The needle enters at (4) and comes out at (3). Point (3) is $4 \mathrm{~cm}$ away from (1) and $2 \mathrm{~cm}$ from cut edge. Point (4) is $4 \mathrm{~cm}$ away from (2) and $2 \mathrm{~cm}$ from cut edge.

d) The two ends of suture being tied in front of linea alba.

e) The small free end of suture pulled inside with an artery forceps or right angle forceps. The small free end of suture tied with long strand of suture. 
f) Not being buried behind linea alba to prevent sinus formation.

g) Two interrupted X-sutures applied $1 \mathrm{~cm}$ apart.

\section{Conventional Continuous Closure}

It was performed using No. 1 Vicryl suture (Polyglactin 910 Johnson \& Johnson Ltd, India), care has been taken to place each bite 1.5-2 $\mathrm{cm}$ from the cut edge of linea alba and successive bites been taken $1 \mathrm{~cm}$ from each other with intermittent locking all along. The edges of linea alba gently approximated without strangulation with an attempt to keep suture to wound length ratio of $>=4: 1$.

\section{Evaluation Parameters}

Operative Time- Time of closure was noted from the start of the closure of abdominal fascia to the close of the abdominal fascia.

\section{Length of Suture Material}

For continuous closure, suture length was calculated by subtracting the length of suture material remaining at the end of closure from the total length of suture taken at the start of procedure. For interrupted-X closure, suture length was calculated by subtracting the length of suture material remaining at the end of closure and the suture lengths that were wasted while dividing suture after tying knots from the total length of suture taken at the start of procedure.

\section{Suture Length to Wound Length Ratio}

The length of the suture material used divided by the length of the incision.

\section{Wound Dehiscence}

Was defined as post-operative missing continuity of the abdominal fascia with bursting open or splitting along sutured lines.

\section{Followup}

Patients were followed up and re-evaluated at 2, 4, and 6 weeks and 3 months after surgery in outpatient department.

\section{The Patients were Examined for following Complications- Wound Infection}

Suture sinus- defined as abnormal protrusion of underlying suture threads through an intact skin, may or may not require removal.

Burst Abdomen/Incisional Hernia- defined as postoperative evidence of a fascia dehiscence after completed superficial wound healing with or without prolapse of abdominal organs.

\section{Statistical Analysis}

The collected data for the above study has been statistically analysed with chi-square and t-test with the help of SPSS software.

\section{RESULTS}

The study included 40 patients, who were divided into two groups.

\section{Age and Gender Distribution}

The mean ages in the two groups 1 and 2 were 35.40 (SD = 15.65) yrs. and 29.60 (SD $=9.15$ ) yrs. respectively. Patients between age group 15-75 were included in the study. Majority of the patients were male 27 (67.5\%).

\section{Time Taken for Closure of Rectus Sheath}

The mean time taken for closure of rectus sheath in group 1 i.e. in the interrupted - X group was $20.30 \mathrm{~min}$. and for closure of rectus sheath in group 2 i.e. the conventional continuous group was 14.05 min. respectively (Table 1). The difference was statistically highly significant with $\mathrm{p}<.001 ; \mathrm{t}=19.109 \mathrm{using}$ independent $\mathrm{t}$ sample test.

\begin{tabular}{|c|c|c|c|c|}
\hline Group & N & Mean \pm SD & 't' value & P value \\
\hline INT-X & 20 & $20.30 \pm 1.17$ & 19.109 & $<0.001^{* *}$ \\
\hline CC & 20 & $14.05 \pm 0.87$ & & \\
\hline \multicolumn{5}{|c|}{ Table 1. Mean Time taken for Closure } \\
\hline
\end{tabular}

${ }^{* *} \mathrm{p}<0.001$; Highly significant.

\section{Suture Length}

Mean length of suture used to close the abdominal aponeurotic sheath in interrupted-X group was $105.30 \mathrm{~cm}$ and in continuous group was $72.45 \mathrm{~cm}$ respectively using similar No.1 Vicryl sutures (Table 2).

\begin{tabular}{|c|c|c|c|c|c|}
\hline Group & $\mathbf{N}$ & \multicolumn{2}{|c|}{ Mean \pm SD } & 't' value & P value \\
\hline INT-X & 20 & 105.30 & \pm 12.62 & 11.122 & $<0.001^{* *}$ \\
\hline CC & 20 & 72.45 & \pm 3.90 & & \\
\hline \multicolumn{6}{|c|}{ Table 2. Mean Length of Suture Material Used } \\
\hline
\end{tabular}

\section{Suture Length: Wound Length (SL: WL)}

SL: WL was calculated by dividing the length of the suture used in closing the rectus sheath and then dividing it by the total incision length of individual patients and then calculating the mean. The mean SL: WL for interrupted-X suturing was 6.09 as compared to 4.31 for continuous suturing. The difference is highly statistically significant between the above two techniques $(\mathrm{t}=18.839$; $\mathrm{p}<0.001)$ using t- test (Table 3).

\begin{tabular}{|c|c|c|c|c|}
\hline Group & $\mathbf{N}$ & Mean \pm SD & ' $\mathbf{t}$ 'value & P value \\
\hline INT $-X$ & 20 & $6.09 \pm 0.298$ & 18.839 & $<0.001^{* *}$ \\
\hline CC & 20 & $4.31 \pm 0.297$ & & \\
\hline \multicolumn{5}{|c|}{ Table 3. Mean SL: WL } \\
\hline
\end{tabular}

${ }^{* *} \mathrm{p}<0.001$; Highly significant.

\section{Total Duration of Hospital Stay}

The mean duration of hospital stay for the interrupted-X and continuous group was 14.65 days and 12 days respectively. The difference was not statistically significant.

\section{Wound Dehiscence}

Wound dehiscence was noted and recorded in interrupted-X and continuous groups in the immediate post-operative period till the time of discharge. Wound dehiscence occurred in $20 \%$ of the total patients. Evaluating patients in interrupted-X group wound dehiscence rate was found to be $15 \%$ as compared to $25 \%$ in the continuous group using same sutures $(p=0.429)$ using chi-square test. 


\section{Burst Abdomen/Incisional hernia}

All patients were evaluated for burst abdomen till 3 months. The burst abdomen rate was more in conventional continuous method of closure. Incidence of incisional hernia in the two groups was $5 \%$ and $15 \%$ respectively at the end of 3 months. In total, the incidence of incisional hernia was $10 \%$. No statistically significant difference was observed while comparing continuous technique with interrupted in terms of burst abdomen/incisional hernia $(p>0.05)$ (Table $4,5,6)$.

\section{DISCUSSION}

The best method of abdominal closure is one that maintains tensile strength throughout the healing process with good tissue approximation, does not promote wound infection or inflammation, is well tolerated by patients and is technically simple and expedient. The specific technique used in closure of the abdominal fascia for the individual is frequently based on non-scientific factors. Because of difficulties arising from differently tailored study designs, the surgical literature has not clearly demonstrated an optimal technique to close abdominal fascia, especially in emergency settings, as patients taken up for elective surgeries are far better prepared to bear the procedure.

In this study, it was observed that abdominal incisions closed with interrupted-X technique though took more time and suture material to be closed in comparison to conventional continuous technique, but development of wound dehiscence, burst abdomen and incisional hernia was far less with the former technique as compared to the latter. So, keeping in view the above, interrupted-X technique should be the preferred method for closing laparotomy incisions. Also, it should be noted that there is increased propensity of chest infections due to burst abdomen as patients are not able to generate the required intraabdominal pressure to expectorate the secretions. This further increases wound pain, length of hospital stay, and cost of care in the form of wound care and sometimes ventilatory support.

\section{CONCLUSION}

1. Interrupted-X closure is better than continuous closure in terms of preventing occurrence of burst abdomen/incisional hernia.

2. Interrupted-X suturing is comparable to conventional continuous suturing in terms of occurrence of postoperative complications of laparotomy wounds like seromas, wound infection, wound dehiscence, hospital stay, and finally burst abdomen/incisional hernia.

3. No statistical difference is present between the occurrence of wound dehiscence and incisional hernia formation between continuous and interrupted-X method of closure.

4. Interrupted-X closure thus becomes the preferred method for abdominal fascia closure for exploratory laparotomies.

\section{REFERENCES}

[1] Riou JP, Cohen JR, Johnson H. Factors influencing wound dehiscence. Am J Surg 1992;163(3):324-30.

[2] Diener KM, Sabine V, Jensen K, et al. Elective midline laparotomy closure: the INLINE systematic review and meta-analysis. Annals of Surgery 2010;251(5):843-56.

[3] Pearl ML, Rayburn WF. Choosing abdominal incision and closure techniques: a review. J Reprod Med 2004;49(8):662-70.

[4] Riet VM, Steyerberg EW, Nellensteyn J, et al. Metaanalysis of techniques for closure of midline abdominal incisions. Br J Surg 2002;89(11):1350-6.

[5] Agrawal CS, Tiwari P, Hadke NS, et al. Interrupted abdominal closure prevents burst: randomized controlled trial comparing interrupted-X and conventional continuous closures in surgical and gynecological patients. Indian J Surg 2014;76(4):2706. 